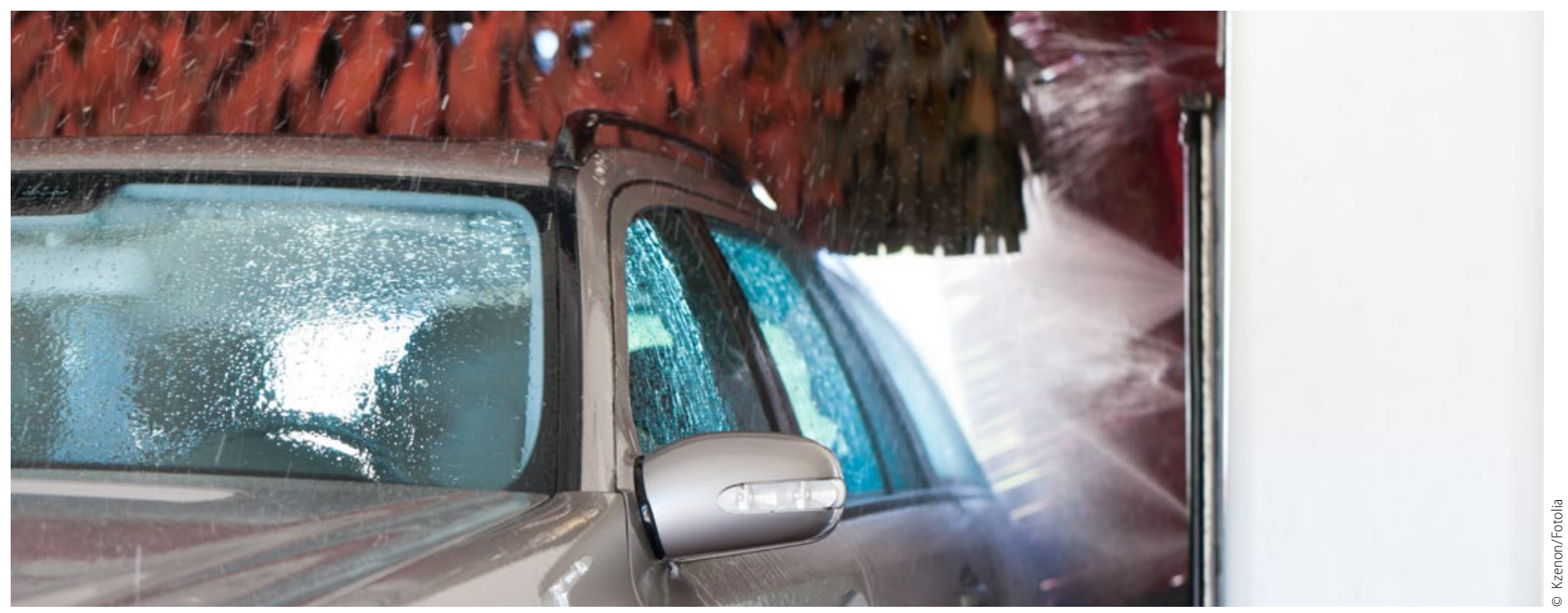

\title{
Auch besondere Umstände machen PZR nicht erstattungsfähig
}

\author{
Die professionelle Zahnreinigung (PZR) ist vom Leistungskatalog der gesetzlichen Krankenversi- \\ cherung (GKV) bekanntlich nicht umfasst. Das gilt auch dann, wenn sie nicht allein einem pro- \\ phylaktischen Zweck dient, wie das Landessozialgericht (LSG) Baden-Württemberg in einem \\ jetzt veröffentlichten Beschluss vom Juli 2015 entschied.
}

\section{Der Fall}

Der Kläger hatte in einer Universitätsklinik auf eigene Kosten zweimal eine PZR durchführen lassen und beantragte wenige Tage später die Erstattung der Kosten bei seiner GKV. Zur Begründung führte er aus, die bei ihm diagnostizierte Parodontitis habe eine PZR ergänzend zur Entfernung weicher und harter Beläge erfordert, da sich anderenfalls die Zahnfleischentzündung massiv verschlimmert hätte. Aus diesem Grund habe es sich um eine zahnmedizinisch notwendige Therapie, nicht um eine Maßnahme der Individualprophylaxe gehandelt. Auf die notwendige Krankenbehandlung habe aber jeder gesetzlich Versicherte einen Anspruch nach dem Fünften Buch Sozialgesetzbuch (SGB V). Die Krankenkasse des Klägers lehnte die Kostenerstattung wie auch die Übernahme der Kosten zukünftiger Zahnreinigungen $\mathrm{ab}$.

\section{Die Entscheidung}

Die Ablehnung geschah zu Recht, wie das LSG Baden-Württemberg auf die entsprechende Klage des Versicherten rechtskräftig entschied. Abgesehen davon, dass der Kläger den Antrag auf Kostenübernahme vor der Selbstbeschaffung der Leistung hätte stellen müssen, stehe dem gesetzlich Versicherten ein Anspruch auf Übernahme der Kosten einer PZR generell nicht zu.

Die PZR sei in den Bewertungsmaßstab für zahnärztliche Leistungen (BEMA-Z) nicht aufgenommen worden. Die Beschränkung der Ziffer 107 BEMA-Z auf das Entfernen von
Zahnbelägen sei bewusst erfolgt und entspreche der einschlägigen Richtlinie des Gemeinsamen Bundesausschusses (G-BA). Dass die PZR nicht in den BEMA-Z aufgenommen worden sei, gefährde auch nicht generell die ambulante zahnärztliche Versorgung. Insofern sei zu berücksichtigen, dass die GKV nicht alle Leistungen erstatte, die medizinisch notwendig, sondern nur diejenigen, die unter Beachtung des Wirtschaftlichkeitsgebots vertretbar seien.

Auch liege keine positive Empfehlung des G-BA zum therapeutischen Nutzen der PZR bei Parodontitis vor, die bisher nur nicht im BEMA-Z umgesetzt wurde. Ein Seltenheitsfall oder ein Systemversagen, in dem auch eine solche Empfehlung des G-BA entbehrlich wäre, sei ersichtlich nicht gegeben.

Nicht zuletzt sei in der Parodontitis auch keine lebensbedrohliche oder regelmäßig letale Erkrankung zur erblicken, die ausnahmsweise die Erbringung einer bisher weder vom G-BA empfohlenen noch im BEMA-Z abgebildeten Leistung zulasten der GKV erfordere.

Damit bleibt es auch bei konkret therapeutischer Zielsetzung dabei, dass die PZR durch die GKV nicht erstattet wird, es sei denn, die Satzung der betreffenden Kasse sieht ausnahmsweise etwas anderes vor. Für den Zahnarzt bedeutet das, dass auch im Fall der therapeutischen Notwendigkeit einer PZR der Patient im Rahmen der sogenannten wirtschaftlichen Aufklärung über seine Kostentragungspflicht zu unterrichten ist.

RA Michael Lennartz, www.heilberuferecht.eu 